\title{
Implementation of Resource Integration and Application of Grouped Mobile Devices Using Wi-Fi Direct
}

\author{
I-Hsuan Peng, Yen-Chieh Lee, Kuang-Lun Yeh \\ Dept. of Computer Science and Information Engineering \\ Minghsin University of Science and Technology \\ Hsinchu, Taiwan
}

\author{
Pin-Man Huang \\ Department of Communication Engineering \\ National Central University \\ Taoyuan, Taiwan
}

\begin{abstract}
Following the constant improvements in technology and rapid developments in the computers, communications, and consumer electronics $(3 \mathrm{C})$ industry, mobile devices have become assistive tools for everyday life. They are lightweight, thin, and convenient for carry and their functionality is akin to a portable computer. However, these devices are normally equipped with small displays. At times when a larger screen is needed, such as when sharing information, watching videos, or discussing in groups of people, the physical device must be passed between all members in the group. Therefore, this study proposes a convenient way to integrate the resources, including display, computation, communication, etc., of multiple mobile devices to create a portable cloud for various applications. This study focused on display integration, combining multiple device screens into one large screen for data display, interaction, and sharing in meetings, for entertainment, or at school. The screens of mobile devices in different locations can also be integrated to create a dynamic billboard. This study also incorporated near field communication (NFC) to share data from one device with all other integrated devices. Wi-Fi Direct was used for group device communication to eliminate the need of Internet connection.
\end{abstract}

Keywords—portable cloud; resource integration; mobile device; Wi-Fi Direct

\section{INTRODUCTION}

Mobile devices are widely used in everyday life, but their displays are small to increase portability. As such, users may feel something is lacking from their devices. For example, resolution may be unclear when viewing photos, such that users must zoom in and view them in sections to clearly see the entire picture. When sharing between friends, the small screen also prevents more than one or two people to simultaneously view the device. If users wish to discuss a problem in a group, it would be impossible for all group members to share a small screen. And this also lacks a convenient method to record key discussion points. The demands people have for mobile devices have expanded from phone calls and text messages to desiring it to be able to handle everything. However, display and other resources provided by mobile devices are limited (resolution, screen size, computational ability, memory, etc.) and there has yet to be any developments or new propositions. This study provides a new method of thinking in order to integrate the resources in a group of devices to create a portable cloud to be used for multiple applications. The combined resources may be any aspect of the device, including displays and computational resources. Information can be shared across the individual screens can as one large screen or as separate screens, and the computational ability of a single device can become part of a multitasking, parallel processor.

\section{RELATED TECHNOLOGIES AND APPLICATIONS}

\section{A. Wi-Fi Direct}

$\mathrm{Wi}-\mathrm{Fi}$ is a form of short-range, wireless transmission technology used in a wide variety of applications; nearly all electronic devices use $\mathrm{Wi}-\mathrm{Fi}$, including personal computers (PCs), cell phones, laptop computers, and multiple embedded systems. Wi-Fi has two models of communication: infrastructure and ad-hoc. In the infrastructure model, all equipment must be connected to an access point which connects other wireless network equipment or access network resources. The ad-hoc model is a network structure without an access point. An ad-hoc network allows Wi-Fi devices within a short range to connect peer-to-peer without relying on an access point. The number of connected devices, the distance between them, and their movement all affect network efficiency; thus, it is more suitable for use in smaller environments. The lack of additional hardware reduces the cost of use; however, the current ad-hoc mode provided by general Wi-Fi is difficult to set up. As each manufacturer's methods differ, very few people currently use ad-hoc networks. Thus, in October 2009, the Wi-Fi Alliance proposed Wi-Fi Direct [1] in the hopes of allowing a direct connection similar to an ad-hoc network with a simplified and standardized setup. This study used Wi-Fi Direct as it is more suited for situations where it is impossible or inconvenient to install network equipment and a quick combinational network is required. The grouping and use of the multiple smart devices in this study may provide a method to easily combine handheld electronic devices when necessary. This requires high mobility and, as such, Wi-Fi Direct was the optimal method of choice.

\section{B. Near Field Communication}

Near field communication (NFC) [2] is a close-range, wireless communications technology that allows for non- 
contact point data transmission between the electronic devices. Developed from radio frequency identification (RFID), NFC is widely used in applications such as electronic payments [3] and the exchange of business cards and address book information. For communication, one device acts as the initiator (starting the communication) and the other device operates in target mode (waiting for the initiator). Any NFC device can act as an initiator or target. In general, all NFC devices begin in target mode and periodically switch to initiator mode to scan the proximity for NFC targets before switching back to target mode. If an initiator finds a target, it sends an initiation sequence to establish communication and then begins to transmit data. NFC can operate in two modes: passive and active. In passive NFC mode, only the initiator provides a carrier field which the target devices accept to produce energy for data transmission without the need for an electrical supply. In active NFC mode, both initiator and target devices require power supplies and communicate by alternately generating their own RF fields. Compared to the passive mode, this mode allows communication over greater distances at higher speeds. Most smartphones today are capable of NFC. NFC was used in this study for data exchange and sharing.

\section{Similar Applications}

Ohta and Tanaka [4, 5] proposed using a "pinching gesture" to associate multiple touch-screen devices. This type of user interface uses a unique application design to instantly connect and break integrated devices. Selmer Nielsen et al. [6] developed the JuxtaPinch software that also uses a pinching gesture to connect multiple touch screen devices in order to share and view pictures and interact (such as drawing on pictures). In this study, devices were combined by drawing a line or selecting a position so that integration can be completed with a single finger allowing data to be displayed separately.

\section{SYSTEM ARCHITECTURE AND PROCEDURE}

The proposed procedure can be subdivided into three steps as shown in Figure 1.

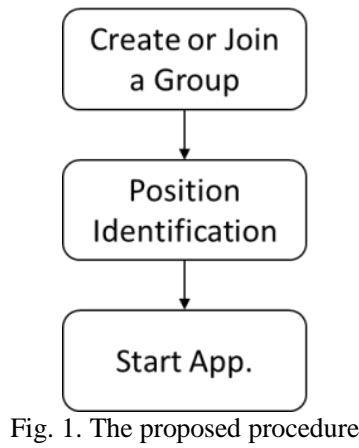

The first step is a device perform neighbor discovery and then choose to join a group or create a group with one neighbor device. Then the second step provides method to configure the position of the combined screens. After the completion of position identification, the device can be added to perform the group application. In order to describe the operation procedure of the proposed scheme, Figure 2 shows an example with 4 mobile devices. The mobile devices with Wi-Fi Direct interface can be integrated regardless of make, size, or operating system. Wi-Fi Direct communication creates a cluster, verifies device positioning, and then can run different applications according to the situation. In Figure 2(a), four devices are used as one large device that can be used to display data that may be retrieved from the Internet or saved on one of the devices. NFC can also be used to transmit data from another device to be displayed on the composite screen. However, if NFC is used, at least one of the devices that make up the composite screen must have an NFC interface. As illustrated in the figure, the cell phone can transmit data to the nearby devices. Figure 2(b) shows a dynamic billboard where devices can be situated in different locations and display a portion of the data.

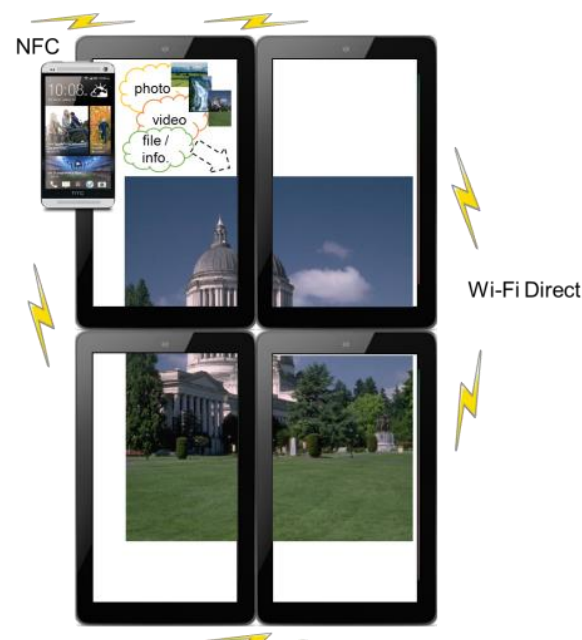

(a)
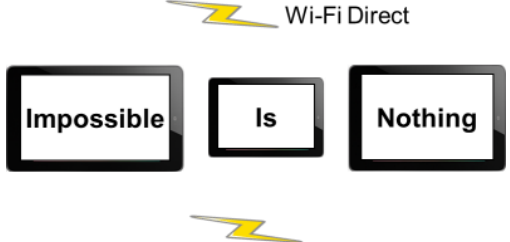

(b)

Fig. 2. System architecture

The device positions in this study were distinguished using two methods. For the first method, the user draws the cross line over the screens so that the devices recognize their positions, i.e. left/right or top/bottom of the complete screen. Where the line ended on the first device and began on the second device alerted the devices of their relative positions (Figure 3(a)). Devices were not required to be physically placed align to each other to use this method. The second method selected the position of the device in the group (Figure 3(b)). Before using this method, users must first designate the arrangement of the devices; for example, all devices in one row or column, or define maximum number of devices in one row or column but can have multiple rows or columns. After the group arrangement is defined, the position must be 
selected on each device in the group. In Figure 3(b), the first device selects position one, the second selects position two, etc. Devices must be placed aligned to use this method; the point of alignment may be the center of each device or others.
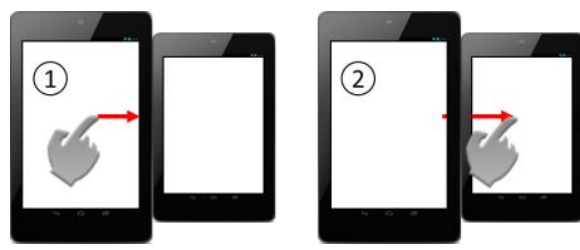

(a)

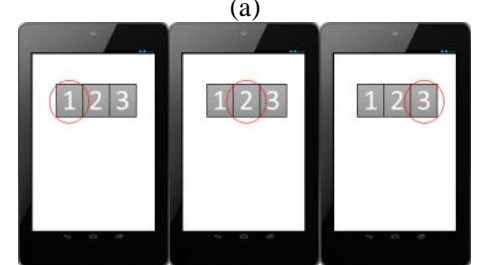

(b)

Fig. 3 Position location in grouped devices

Regardless of the method used, the devices' screen size and resolution must be input when defining their relative positions for consistent display of data. Application software can recognize the clustered devices as multiple display panels to display different content or as one large display panel to display one large image. The coordinates given to a device when touching one of the screens will be converted into coordinates for the larger display panel according to the position of the device in the arrangement. As each device is aware that it is part of a larger screen as well as its relative position, users can select and draw on the screens to operate the clustered devices as if it were one large screen device.

As Wi-Fi Direct was used, one of the devices used must be designated as the coordinator (group owner) while the others are clients. The coordinator is selected automatically according to computational ability when initiating the Wi-Fi Direct link. When grouping, the surrounding area can be scanned for devices with active Wi-Fi Direct and the desired devices can be selected and added to the group

\section{IMPLEMENTATION RESULTS}

Figure 4 shows a group of devices organized in a single row. Behind the first device is an NFC enabled device. Current NFC transmission between mobile devices requires the backs of the two devices to be touching; thus, it was necessary to place this device underneath. During data transmission, the application program will be reduced in size (Figure 4). Figure 5 shows data being moved through multiple devices after transmission was completed. Data browsing and transmission is allowed in this setup; if sharing a picture with multiple people, the devices do not need to be closed together. This application allows more than displaying images and videos; any information can be displayed. For example, at a musical concert or other situations, the grouped devices can be used to display a sentence with one or more words shown on each screen as determined by their size and resolution, so that the performer can see it clearly.

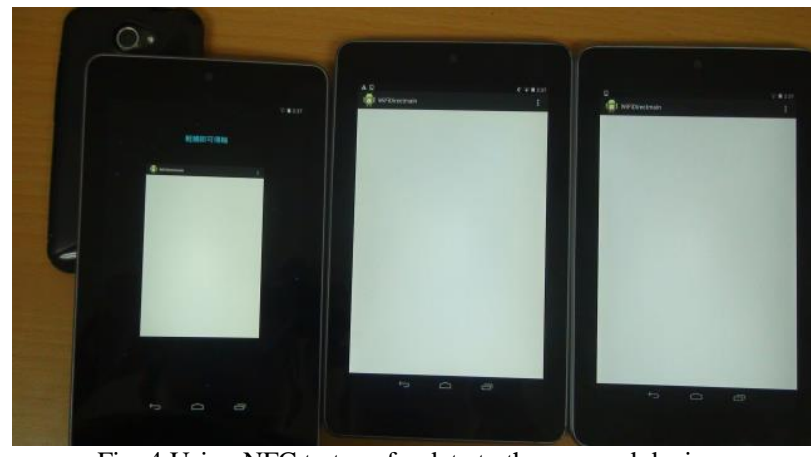

Fig. 4 Using NFC to transfer data to the grouped devices

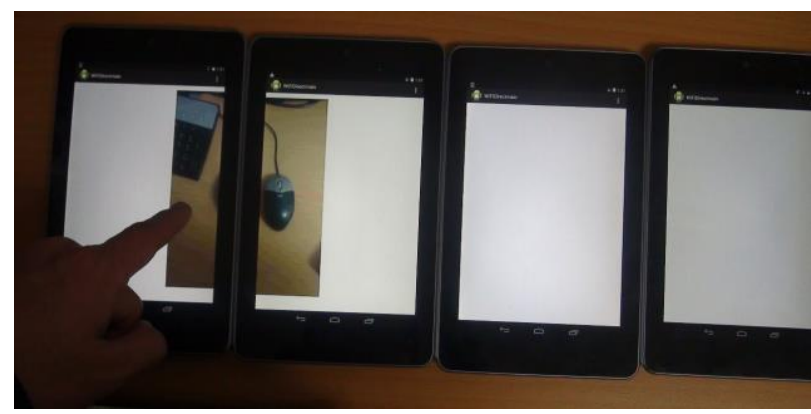

Fig. 5 display picture in grouped devices (one row)

Figure 6 shows a group with multiple rows where multiple images are displayed across the entire composite screen as the bottom two devices. Photos can also be spanned between multiple devices, as shown in Figure 6 intermediate.

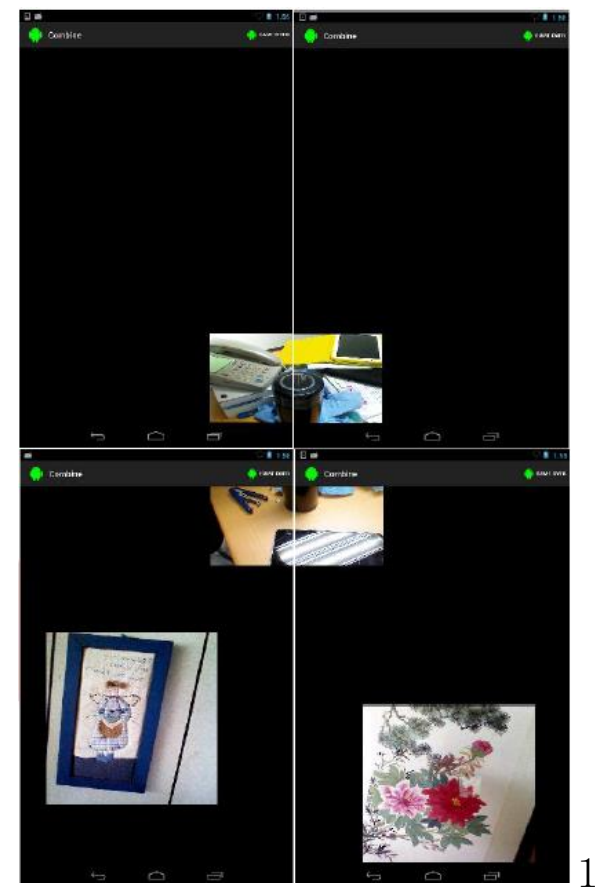

Fig. 6 display picture in grouped devices (multiple rows)

\section{CONCLUSIONS}

This study clustered mobile devices using Wi-Fi Direct, successfully integrating resources and creating a portable 
cloud. Resource integration was completed using touchscreen devices and two applications were proposed. The first was a composite screen made up of multiple devices for data transmission and sharing; the second was a dynamic billboard that dispersed information across the clustered devices.

Future studies can research and develop further concepts and improvements. For example:

- A portable electronic table: traditional electronic tables (such as Microsoft's PixelSense [7]) are expensive to manufacture, large in size, and remain fixed in one location. The novel application presented in this study can combine existing devices while allowing transmission of data from an outside NFC enabled device to create an electronic table that is inexpensive and lightweight.

- An electronic whiteboard: traditional whiteboards and electronic whiteboards are often only found in conference rooms. When discussions take place outside of a conference room, most people may simply use a piece of paper to aid in explanations. This is not conducive for discussions and information can be easily lost. The methods proposed in this study to group devices can be used anywhere for instantaneous sharing and editing and allows quick and easy saving of discussion content and transmission of data files.

- A tabletop gaming platform: traditional tabletop games require game pieces; any game can be developed for this platform, such as mahjong and card games, to satisfy demand in the tabletop game market.

- For applications similar to the dynamic billboard, clustered devices may be randomly distributed throughout a venue. Device locations can be determined using technologies such as iBeacon [8] and Bluetooth Smart [9] for automatic recognition of locations.

\section{ACKNOWLEDGMENT}

This research work was supported in part by the grants from the Ministry of Science and Technology (grant number: MOST 103-2221-E-159 -003).

\section{REFERENCES}

[1] "Wi-Fi Direct |Wi-Fi Alliance", http://www.wi-fi.org/discover-wi-fi/wi-fi-direct

[2] "NFC Forum", http://nfc-forum.org/

[3] A. Bodhani, "New ways to pay", IET Engineering \& Technology, Vol. 8, Issue 7, pp. 32-35, Aug. 2013.

[4] Takashi Ohta and Jun Tanaka, "Pinch: An Interface That Relates Applications on Multiple Touch-Screen by 'Pinching' Gesture", Proc. of the 9th Advances in Computer Entertainment Conference (ACE 2012), volume 7624 of Lecture Notes in Computer Science, pp. 320-335, Springer, 2012.

[5] Takashi Ohta and Jun Tanaka, "Using Pinching Gesture to Relate Applications Running on Discrete Touch-Screen Devices", International Journal of Creative Interfaces and Computer Graphics archive, Vol. 4 Issue 1, pp. 1-20, Jan. 2013.
[6] Heidi Selmer Nielsen, Marius Pallisgaard Olsen, Mikael B. Skov and Jesper Kjeldskov, "JuxtaPinch: An Application for Collocated MultiDevice Photo Sharing", Proceedings of the 16th international conference on Human-computer interaction with mobile devices \& services, Sept. 23-26, 2014, Toronto, ON, CA, pp. 183-192.

[7] "Microsoft PixelSense", http://www.microsoft.com/en-us/pixelsense/defaultaspx

[8] "iBeacon for Developers - Apple Developer", https://developer.apple.com/ibeacon/

[9] "Bluetooth Smart (Bluetooth Low Energy)", http://www.bluetooth.com/Pages/Bluetooth-Smart.aspx 